\title{
Break Verbs in Caused-motion Construction
}

\author{
Xiaorong Xia \\ School of Foreign Languages and Cultures, Nanjing Normal University, Nanjing, Jiang Su Province, China \\ Email: xiaxiaorong1644@ sina.com
}

\begin{abstract}
The relation between verbs and constructions has been the focus of linguistic research. It is generally believed that verbs are more likely to occur in constructions of the similar sense. Break verbs encoding change of state can appear in caused-motion construction, however. From a connectionist view with the framework of conceptual frame, an indirect connection between break verbs and the caused-motion schema can be established, and verbs occur in the construction as a result. Moreover, certain semantic features connect some verbs more closely to the construction.
\end{abstract}

Index Terms - break verbs, caused-motion construction, a connectionist view

\section{INTRODUCTION}

A verb may participate in different syntactic structures and new senses arise as a result. In the following sentences, the verbs break, run, laugh, and shout appear in the same construction and have the new sense of causing something or somebody to move on a path.

(1) a. He threw the ball into the hole.

b. He put an apple on the table.

c. He broke the walnuts into the bowl.

d. They ran the rats through the maze.

e. We laughed him out of the room.

f. He shouted her out of the room.

Compared to caused-motion verbs (like throw and put), these verbs in isolation, however, lack this caused-motion sense. Accordingly, their argument structures are inconsistent with the semantic structure of the sentences where they occur. From a projectionist view, the verbs fail to provide semantic interpretation for the arguments in the sentence. In order to explain the semantic relation between the verb run in (1c) and the syntactic arguments, it is necessary to posit a different argument structure for the verb, namely, run <agent, runner, path>. Verbal polysemy thus occurs, but it is not favored by parsimony.

Goldberg's (1995) probe into caused-motion constructions sheds new light on the research. She chooses to interpret caused-motion in terms of a construction combining verb and directional preposition to yield a particular, conventionalized interpretation.

"Such a construction must be specified in grammar to account for certain cases in which the semantic interpretation cannot plausibly be attributed to the main verb and other means of deriving the semantics compositionally also fail" (Goldberg, 1995, 152).

Goldberg (1995) assumes that caused-motion semantics doesn't exist in any of lexical items independently, but in what she calls constructions. As a result, causative or motion explanation of the verbs in the sentences (1c-1f) arises from constructions, while the basic senses of the verbs remains. Whether the verbs are typical caused-motion verbs like throw or put, or the ones that lack path argument, cause argument or theme argument, they gain caused-motion interpretation from the construction.

Goldberg's caused-motion argument structure construction is very attractive in that it licenses the non-subcategorized arguments while avoiding additional senses of a verb. This syntactic-semantic generalization of caused-motion has been embraced by many cognitive researchers. Kay (2002, 2005), Iwata (2005), Boas (2003), Langacker (2005), agree on such a caused-motion construction. Basing on Goldberg's research, they take further study into the construction.

However, Goldberg's constructional argument structure also faces some problems. Caused-motion argument structure construction is supposed to provide a caused-motion sense to non-caused-motion verbs with the fusion between constructional argument roles and verbal participant roles. Fusion, according to Goldberg (1995), elaborates the relation between verbs and the construction. However, fusion does not explicate why some verbs can fuse into the caused-motion construction while others are denied. In addition, not all verbs of the same class can fuse with the construction. So what encourages or prevents the use of verbs in the construction is not expressed by fusion.

Break verbs, encoding change of state, are not supposed to appear frequently in caused-motion construction. Goldberg (1995) posits that change of state is a metaphor of change of position. The appearance of break verbs in caused-motion construction indicates the productivity of the construction, in that it can attract verbs of quite different senses. On the other hand, there must be some semantic features that facilitate the appearance of break verbs. Furthermore, some appear more frequently. 


\section{BREAK VERBS}

The corpus data in WebCorp (http://www.webcorp.org.uk/) show that "snap" and "smash" are more frequently used in caused-motion construction than other verbs in the class. The verbs "fracture" and "splinter" appear much less than other verbs. Most verbs show high collocation strength with certain prepositional types. The most distinctive is the use of "crack" with goal type prepositions. And even amazing is the repetition of "egg" as the direct object, amounting to 18 times in the total 21 caused-motion uses found. The caused-motion sense of these sentences is scene-specific. The crusts of eggs are cracked and the egg yolk and egg white fall out of eggs into the vessels or mixtures. The windower of the scene catches two discontinuous subevents---the initiator's cracking subevent and the mover's final position--- and establishes a causal relation between them.

The data also show that "crack", "chip" and "break" have relatively more uses with "out of", and the first two verbs, together with "smash", "crush" and "crash", are also more frequently found with goal type prepositions. The verbs "smash" and "crash" even appear more with trajectory type prepositions. The verb "snap" is the only one that can be used with the three types of prepositions.

Except "crash", the other break verbs have more occurrences with source type prepositions than those with goal type prepositions. The sense of breaking something appears nearly exclusively in the instances with source prepositions from, out of and off. But a few verbs, "snap", "smash", "chip", "crack" and "crush", mean similar to "cause to move", "hit" or "compress", but not similar to "break". In the following sentences, the affected undergoes change of location because of force exerted on it.

(2) a. you need to smash the stone from the shelf

b. possession of the ball will snap the football from the nearest hash mark

c. differential pressure just to crack the ball off the seat

d. who used to crush the ball off the tee/ability to crush the ball from the baseline

e. The puck carrier tries to chip the puck off the board into neutral zone.

Apparently, most break verbs can be used in caused-motion construction. Goldberg (1995) sets a semantic constraint on the use of break verbs in caused-motion construction. A definite effect on the theme arguments other than motion is implied in the following sentences because they change the shape as a result of the action denoted by these verbs.

(3) a. The butcher sliced the salami onto the wax paper.

b. Joey clumped his potatoes into the middle of his plate.

c. Joey grated the cheese onto a serving plate.

d. Sam shredded the papers into the garbage pail.

But Goldberg (1995) points out that these verbs of change of state are different from strike verbs, which also entail a definite effect on the theme argument, in that they denote the action which implies some predictable incidental motion. For example, when we slice the salami we may arrange for wax paper for the sliced salami to fall onto it; meanwhile, we would slice the salami in the direction of the wax paper. It is the path of this predictable incidental motion that can be specified by the directional phrase.

The act denoted by these verbs indicates conventional scenarios which allow them in caused-motion construction. The motion of (4b) is intended and thus a conventional motion. But it is not the case with (4a).

(4) a. * Sam unintentionally broke the eggs onto the floor.

b. Sam carefully broke the eggs into the bowl.

So Goldberg (1995) contends that "If the activity causing the change of state (of effect), when performed in the conventional way, effects some incidental motion and, moreover, is performed with the intention of causing the motion, then the path of motion may be specified"(p.172). With a caused-motion argument structure construction, Goldberg and her followers would insist that the senses of these verbs of change of state would remain unchanged and they inherit the caused-motion sense from the constructional argument structure, which licenses the path unsubcategorized in the lexical senses. But why the constructional argument structure licenses the use of "break" in (4a) but not that in (4b) involves the judgment by means of conventional scenario. That is, the acceptability of (4a) and the unacceptability of (4b) depend more on our world knowledge, but not only on lexical or constructional argument structure.

\section{A CONNECTIONIST VIEW}

Conceptual frame advocated by Cheng (2006) represents a gestalt of encyclopedic knowledge or experience and aims to describe clauses by formalizing their conceptual semantics and relating the conceptual contents expressed by the clauses. The conceptual frame of a clause is equal to its schema, which is described by different conceptual structures. According to Cheng (2010), semantics should be related to the perception system if human beings can express verbally the event they see or perceive. Similarly, if human beings can act according to what they are told, then the semantics should also be related to the motor system. Conceptual frame, as a part of the whole conceptual semantic system, should be realized into syntactic structure on the one hand, and on the other hand provide necessary conceptual information for cognitive operation (such as questions, answers, semantic reasoning, etc.) (Cheng 2006). Semantics serves as the interface between sensory-motor structures and morphosyntactic structures (Cheng 2010).

The conceptual frame of a caused-motion instance includes two conceptual structures: an action conceptual structure 
describing the action denoted by verbs, and a causative location conceptual structure depicting the schema of the sentence. As Figure 1 shows, the throwing action is similar to the caused-motion schema and thus the components of the action conceptual structure conflate with those of the causation conceptual structure. In other words, the throwing action is connected directly to the caused-motion schema so that the verb is conventionally used in caused-motion construction.

\begin{tabular}{|l|}
\hline He threw the ball into the hole. \\
\hline Actor $_{\text {he }}+$ Act $_{\text {throw }}+$ Acted $_{\text {ball }}+$ Goal Location $_{\text {into the hole }}$ \\
Causer $_{\text {he }}+$ Cause+ Object \\
ball + Goal Location \\
Figure the hole
\end{tabular}

Break verbs are not conventionally used in caused-motion construction; however, indirect connection may be built only if their action conceptual structure and causation conceptual structure can be mediated by certain conceptual contents. The examples above demonstrate that it is necessary that action denoted by verbs of change of state should entail motion; otherwise, it cannot be connected to caused-motion event. That is to say, whether verbs of change of state can express a caused-motion sense depends on the conceptualizer's attention. If it extends to the motion subevent as a result of change of state, the action involving change of state would be more likely to be construed into a caused-motion semantic structure.

As Figure 2 shows, there are three extended conceptual structures between action and caused-motion event. The first describes the change of state of Acted "walnuts"; the second is a creation conceptual structure, indicating that the breaking action creates "nutlets". Anyway, we aim to get nutlets by means of breaking walnuts. The third conceptual structure depicts the change of location of the created. They mediate between action conceptual structure and causation conceptual structure in the conceptual process to connect them. The breaking action brings about change of state of Acted, which in turn leads to the occurrence of Created, which undergoes change of location. The connection between action and event is achieved by the three conceptual structures encoding cause and effect.

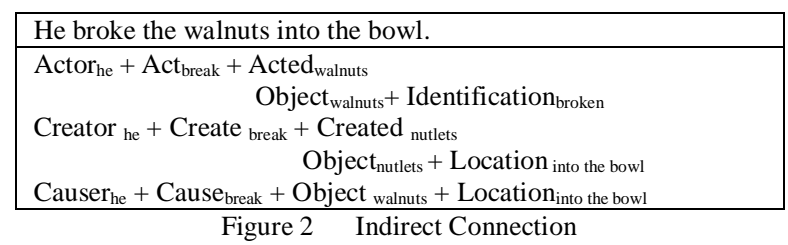

That is to say, the use of break in caused-motion construction needs the construal of extensions. The extended sense is not encoded in the break event but inferred from the specific scene of breaking walnuts. In contrast, motion is hardly construed in the case of breaking the window, even though glasses may fall out of the window onto the floor. The indirect connection between the breaking action and caused-motion schema involves world knowledge. This explains the creative uses of most break verbs in caused-motion construction.

According to the connectionist view, verbs can creatively occur in certain syntactic structures if connection can be built between action denoted by verbs and events expressed by syntactic structures. The connectionist view within conceptual frame can not only explain why some verbs are conventionally used in certain constructions, but also why some can be creatively used while others are denied from participation into the constructions. Only when there is a connection between verbs and constructions can we say verbs would fuse into constructions.

\section{Semantic Features}

As verbs of change of state, break verbs have the sense quite different from that of caused-motion construction. The occurrences of caused-motion construction found in the corpus data of these verbs just stress the productivity of the construction. Break verbs do have a relatively low token frequency with the construction; however, members of break verbs still present differences when they are used in the construction, because they have subtle differences in lexical senses. The different semantic features make break verbs are connected to caused-motion construction to different degrees.

\section{A. Motion}

Although break verbs necessarily entails change of state, it does not mean that these verbs are not related to motion. Regardless, the change of state is brought about force exertion, which is an essential feature to lead to motion. In fact, some break verbs encode motion in their events.

The verb "crash" is found more often with trajectory type and goal type prepositions. Among the 54 occurrences with these two types of prepositions, 42 instances express a literal caused-motion sense. The verb in these instances means "cause something to move noisily, destructively, or violent" or "cause something (like a car) to strike against something with great force, causing damage or destruction"1. The two sentences in (5) express these two senses. The sense of

\footnotetext{
1 The senses of verbs of change of state all come from MSN Encarta (http://encarta.msn.com/).
} 
"breaking into pieces violently and noisily" is not found in the instances.

(5) a. that she intends to crash the car through the living room.

b. to spill the wine and crash the glasses onto the floor

That is to say, "crash" is used in caused-motion construction because its sense is similar to it. The motion sense has been entrenched in its lexical meaning. The parameters of a crashing event, such as noise, violence, speed, etc., accompany the motion against an entity. Or put it another way, they are part of the motion.

Besides "crash", "snap" and "smash" appear in caused-motion construction with the sense of "causing to move". The 61 instances of all the 71 occurrences of "snap" with trajectory type and goal type prepositions have the sense of "cause something to move quickly and sharply". This sense also appears in the uses with source type prepositions, one with "from" and 13 with "out of".

(6) a. power of the wrist to snap the ball across the table tennis table / who must snap the ball through the air to his signal-caller. the tab

b. and snap the tank toward the vacuum so it is snapped securely / wedge in the opening to snap the spring onto

c. possession of the ball will snap the football from the nearest hash mark/person releases enough power to snap the ropes out of the other's hands/

The breaking sense only occurs when "snap" is used with source type prepositions.

(7) a. And I snap the doors from the hinges.

b. late spring frosts that virtually snap the buds off the vines and steamy

With similar semantic features to "throw", such as, force and motion, "snap", among break verbs, is the most often used in location variant with the three types of prepositions. A larger number of caused-motion instances also occur to "smash" when it is used with trajectory type and goal type prepositions. But the senses of "smash" in the instances range from moving, hitting to breaking.

(8) a. he is going to smash the guitar onto the tree/he would smash the mirror onto the ground

b. stepped inside John Nutter to smash the ball into the wall/and then smash the ball through the rim

c. and smash the breading onto the egg and flour mix/and smash the peppers through the sieve

The sentences in (8a) involve the sense of "cause something to move violently so as to break something". Thus the guitar and the mirror would break into pieces because their motion in contact with the tree and the ground, respectively. The sentences in (8b) can be explained as similar to a hit event. Under the hitting force, the ball moved into the goal or traversed a path.

In the sentences of (8a) and (8b), the change of state is gapped. The sentences of $(8 c)$, in contrast, result from the profile of the change in the identificational domain. The motion takes place after a change of state. It is contrary to the sentences of (8a) and (8b), in which a change of state arises from a motion on a path.

\section{B. Not Fully Apart}

The verb "crack" implies the degree of something being broken: not fully apart. Its appearance in caused-motion construction reveals strong collocation strength. The patient "egg" occurs 11 times in its 11 occurrences with "into" and 8 times in its 11 occurrences with "onto". All the instances express both change of state of eggs and change of position of egg wash.

(9) a. Once you crack the egg onto the sidewalk/Why crack the egg onto the bowl first? / you can actually crack the egg onto the pan directly

b. Crack the eggs into the bowl!/crack the eggs into the skillet/I crack the eggs into the same bowl

Fracture has the same feature of not fully apart and it is even less used in caused-motion construction. It results from a subtler distinction in the lexical sense. That is, "fracture", similar to "splinter", has special requirements for the broken pieces. The former implies the broken bones while the latter usually involves the sharp broken pieces. The special requirement limits their uses and it might have caused the least appearances of these two verbs in caused-motion construction. Anyway, it is hard to imagine our fractured bones would move some place once broken. (10a) and (10b), as a result, only express the change of state of the affected. The prepositional phrases only indicate the location where fracturing and splintering take place. In (10c), however, the appearance of a goal type prepositional phrase is indicative of a caused motion to a goal following the change of state of the cork.

(10) a. attempts to fracture the glass along the furrow line have been unsuccessful.

b. splinter the wood along the door jamb further adding flying debris

c. Be careful not to splinter the cork into the bottle.

\section{Being Apart}

Verbs "tear", "rip" and "chip" predicate breaking one piece or some pieces off something. That might account for the large occurrences of "tear" and "rip" with source type prepositions and all of them involve a source body as location. A more typical caused-motion sense occurs in their instances with goal type prepositions, in which the special manner of breaking brings about motion.

The verb "chip" has the sense of "breaking one or more small pieces from something", but special requirement is imposed on something---something hard or brittle. This requirement makes the use of "chip" similar to cutting or 
chopping something. It is also listed as cut verbs.

(11)eventually a roofer can chip the ice out of the gutters/cut open the gums and chip the tooth out of the bone/I had to chip the coralline out of the pump heads.

(12) chip the shells off the rocks/to chip the bump off the side of her big toe

And this sense does not occur in all the goal instances. The verb in (13) and (14) expresses "hitting something". 14 "onto" instances in fact contain one collocation: "chip the ball onto the green". 7 of 9 "into" occurrences involve ball as the theme in motion.

(13) The goal is to chip the ball onto the green / An iron used to chip the ball onto the green / you were able to chip the ball onto the green

You can just chip the ball into the hole. / allowing the Briton to chip the ball into the empty court/cheekily chip the ball into the empty part of the goal.

The entities chipped are always hard or brittle. It means that chipping needs exertion of greater force than tear event and rip event. This parameter is the compulsory condition of caused-motion schema and thus "chip" inclines to gain a new sense related to exertion of force.

\section{Breaking into Pieces}

The sense of "breaking into pieces" also appears in caused-motion construction, especially when trajectory type and goal type positions are used. 9 caused-motion uses of "smash" with the sense of breaking appear in 21 "onto" instances, and 9 appear in 18 "through" instances (see sentences in (8)).

But the other verbs involving the core feature of "breaking into pieces" are less found to express a caused-motion case. This is related to different manners of breaking something into pieces. Split, similarly entailing the sense of breaking something into pieces, is seldom found to express a caused-motion sense. Among its 28 instances with trajectory type prepositions and 26 instances with goal type prepositions, there are only two caused-motion instances.

(15) you may want to split the photos onto the guests tables/we split the rest onto the 2 tortilla and enjoyed it

The reason might be related to the manner of breaking embodied in the sense of "split". That is, "split" involves breaking something by separating or dividing it lengthwise into two or more parts. The through-phrases in (16a) point to the place where the splitting action is accomplished while the into- and onto-phrases in (16b) indicate separated parts, namely, the created object deriving from the action.

(14) a. you should at least split the logs through the heart line. / To split the thighs through the bone, / taking care not to split the wood through the part left for the handle

b. We split the proof onto the following four steps/where abuse is involved to split the partners onto the good side;/Therefore we can split the sum into the two parts./informally split the party into the Aquino and the Angara wings/Let us split the telescope into the following 3 subsections

Other verbs encoding breaking into pieces, like "break", "tear", "rip", "splatter" and "splinter" have few instances with trajectory and goal type prepositions, considering an extension of location conceptual structure is necessary to connect these verbs to caused-motion construction. That is, the action of breaking something into pieces must involve a subsequent motion. It depends more on what is affected in specific context.

Even in their occurrences with source type prepositions, only the following are found to express the sense of breaking into pieces. The others, as discussed above, express separation of part from body.

(17) a. so as not to shatter the grain out of the husks/shatter the seeds from the stalks

b. You want to basically crush the juice out of the limes/drinkers who crush the juice from the plant

\section{CONCLUSiON}

This paper, basing on the corpus data of break verbs in caused-motion construction, demonstrates that break verbs can occur in the construction by means of construing motion from the basic action conceptual structure. From a connectionist view within the framework of conceptual frame, the paper suggests when indirect connection arises between the breaking action and caused-motion schema, verbs are connected to the construction.

The paper further analyzes the semantic features of break verbs and indicates that some features may facilitate the connection between verbs and the construction while others have less influence. The verbs with the semantic features [+motion] and [+not fully apart] are more connected to caused-motion construction, since they are more semantically similar. In comparison, the verbs with the features of [being apart] and [+breaking into pieces] have less connection to the construction.

The connectionist view holds that verbs are connected to constructions where they appear conventionally, because of their semantic similarity. Break verbs have a different sense from caused-motion construction; as a result, their occurrence in the construction involves world knowledge, which decides whether change of position can be extended from the breaking action. The verbs that have semantic features similar to the construction are more likely to occur in the construction.

\section{REFERENCES}

[1] Boas, Hans C. (2003). A lexical-constructional account of the locative alternation. In L.Carmichael, C.-H. Huang, and V. 
Samiian (eds.). Proceedings of the 2001 Western Conference in Linguistics, Vol. 13, 27-42.

[2] Goldberg, Adele E. (1995). Constructions: A Construction Grammar Approach to Argument Structure. Chicago and London: The University of Chicago Press.

[3] Iwata, Seizi. (2005). The role of verb meaning in locative alternation. In Mirjam Fied and Hans C. Boas (eds.). Grammatical Constructions. Amsterdam/Philadelphia: John Benjamins Publication Company, 101-118.

[4] Kay, Paul. (2002). Patterns of coining. http://www.icsi.berkeley.edu/ kay/coining.pdf (accessed 08/03/2008).

[5] Kay, Paul. (2005). Argument structure constructions and the argument-adjunct distinction. In Mirjam Fied and Hans C. Boas (eds.). Grammatical Constructions. Amsterdam/Philadelphia: John Benjamins Publishing Company, 71-98.

[6] Langacker, Ronald W. (2005). Integration, grammaticization, and constructional meaning. In Mirjam Fied and Hans C. Boas (eds.). Grammatical Constructions. . Amsterdam/Philadelphia: John Benjamins Publishing Company, 157-189.

[7] Qilong, Cheng. (2006). Conceptual Frame and Cognition. Shanghai: Shanghai Foreign Language Education Press.

[8] Qilong, Cheng. (2010). Conceptual Frame and Morphosyntactic Variants. Speech delivered in Wuhan University of Science and Technology.

Xiaorong Xia was born in Nanjing, Jiang Su Province, China in 1973. She received her M.A. degree in linguistics in Central South University, Changsha, Hu Nan Province, China, 2001. She is currently studying for Ph. D degree in Linguistics in Nanjing Normal University, Nanjing, Jiang Su Province, China.

She is a lecturer in School of Foreign Languages and Cultures, Nanjing Normal University, Nanjing, Jiang Su Province, China. Her research interests include theoretical linguistics, cognitive linguistics and semantics. 